\title{
EFFECT OF GENISTEIN AND OXALIPLATIN ON CANCER STEM CELLS IN ORAL SQUAMOUS CELL CARCINOMA: AN EXPERIMENTAL STUDY
}

\author{
Ahmed M. Hussein ${ }^{1} M S C$, Sahar M. El-Sheikh² PhD, Zeinab E. Darwish ${ }^{3} P h D$, Khaled A. \\ Hussein ${ }^{4} P h D$, Ashraf I. Gaafar ${ }^{5} P h D$
}

\begin{abstract}
INTRODUCTION: Squamous cell carcinoma is one of the malignant diseases that affect the oral cavity worldwide. One of the theories regarding oral carcinogenesis is that tumor growth is dependent on cancer stem cells. Markers specific for these cells as CD44 have been investigated in hope of developing a deeper understanding for their role in carcinogenesis. Genistein, as chemopreventive agent, has been shown to suppress the growth of several tumors. Oxaliplatin is a chemotherapeutic compound that did show a range of antitumor activity. OBJECTIVES: This research was carried out to study the effect of genistein, oxaliplatin either alone or in combination during experimentally DMBA induced hamster buccal pouch carcinogenesis using CD44 antibody as a marker.

MATERIALS AND METHODS: A total of 100 young Syrian hamsters distributed into groups as follows: 4 normal animals examined for the histology of the normal pouch mucosa and 96 animals divided into; group I, as a control group, in which pouches were painted with a heavy mineral oil only; group II were painted with DMBA mixed in a heavy mineral oil. These animals were randomly divided into 4 subgroups as following: group IIA only painted with DMBA; group IIB where genistein was orally administrated; group IIC were injected with oxaliplatin; and group IID in which both genistein and oxaliplatin were given.

RESULTS: Both genistein and oxaliplatin provided a significant reduction in carcinogenesis process of DMBA induced oral squamous cell carcinoma. Moreover, they provided a significant decrease in the proliferation and activity of cancer stem cells as measured by the CD44 antibody.

CONCLUSIONS: Genistein provides a chemoprevention role and the oxaliplatin produces a chemotherapeutic effect during the process of carcinogenesis. The combined action of both agents was better than the effect of each agent alone.

KEYWORDS: Genistein, Oxaliplatin, Cancer stem cells, CD44.

1. Assistant Lecturer of Oral and Maxillofacial Pathology Department, Faculty of Oral and Dental Medicine, SVU, Kena, Egypt.

2. Vice Dean of Student's Affair, Professor of Oral Pathology, Faculty of Dentistry, Alexandria University, Alexandria, Egypt.

3. Head of Oral Pathology Department, Professor of Oral Pathology, Faculty of Dentistry, Alexandria University, Alexandria, Egypt

4. Professor of Oral Pathology, Department of Oral Pathology, Faculty of Dental Medicine, Al-Azhar University, Assiut, Egypt.

5.Associate Professor of Oral Pathology, Department of Oral Pathology, Faculty of Dental Medicine, Al-Azhar University, Assiut, Egypt.
\end{abstract}

\section{INTRODUCTION}

Cancer is a serious public health problem in many parts of the world; oral cancer is among the 10 most common cancers worldwide (1). Oral squamous cell carcinoma (OSCC) is the most prevalent malignant lesion representing more than $90 \%$ of oral carcinomas. The prognostic value of histologic classification of conventional OSCC (well, moderate and poorly differentiated types) is controversial, so that some authors now recognize that microscopic classification alone is poorly correlated with outcome and response to treatment (2).

The hamster buccal pouch (HBP) carcinogenesis model is one of the most well characterized animal tumor models used to investigate multistage oral carcinogenesis. The HBP carcinogenesis induced by $7, \quad 12$ dimethylbenz[a]anthracene (DMBA) shows extensive similarities to the human OSCC (3). The DMBA is a potent sites specific carcinogen known to induce multistep carcinogenesis, preceeded by a sequence of hyperplasia, dysplasia and carcinoma. The HBP model offers a number of advantages including a simple and predictable tumor induction procedure, easy accessibility for examination and follow up of lesions. This model can be used to test both chemopreventive and chemotherapeutic agents (4).

To develop more effective therapies for OSCC, it is essential to gain a deeper understanding of the biology of this disease and the cells that are responsible for recurrent and persistent cancer (5). The cancer stem cells (CSCs) theory of carcinogenesis postulates that tissue stem cells or progenitor cells are a target for genetic changes that lead to malignant transformation. Based on their similarity to normal stem cells, CSCs are also likely to be more resistant to cancer therapy and may be responsible for tumor persistence and recurrence (6).

Specific markers for CSCs population have been investigated in the hope of developing a deeper understanding of their role in the pathogenesis of oral carcinomas and providing a novel therapeutic strategies. A commonly employed method for CSCs isolation consists in sorting them based on cell surface marker expression. The most commonly used surface marker to identify CSCs is the CD44 monoclonal antibody (7). CD44 is a cell surface glycoprotein involved in cell adhesion, cell to cell interactions and migration. It is a receptor for hyaluronic acid, through which the tumor cells drive an increase in the proliferation and survival rate. Moreover, the CD44 plays an important role in invasion and metastasis of a variety of tumor cells (8).

Natural products are a rich source of compounds that have many applications in the fields of medicine, pharmacy and biochemistry (9). Chemoprevention is a novel and promising approach to control, inhibit or suppress the tumor promotion by using natural entities. A large number of phytochemicals ingested in human diet represent a chemopreventive agent which possess antimutagenic, 
anticarcinogenic, antioxidant and anti-cell proliferating properties (10).

Genistein is a major isoflavone, it is present in soybean and has exhibited significant chemopreventive activity. The mechanisms by which it exerts its anticancer roles include down regulation of androgen mediated carcinogenesis, decreasing genes related to cell proliferation and induction of tumor cell apoptosis. In addition, genistein has been suggested to inhibit tumor angiogenesis and has also an antioxidant role through its molecular targets $(11,12)$.

Effectiveness of natural chemopreventive agents in combination with anticancer drugs may prove to be a significant advance over conventional chemotherapeutic therapy (13). The latter drugs work in all phases of the cell cycle and are used to treat many different cancers (14). Oxaliplatin is a third-generation platinum based chemotherapy drug that has importance in the treatment activity against various types of cancer. Structurally, it contains a 1, 2-diaminocyclohexane carrier ligand that enhances its antitumor activity. It forms intrastrand links between 2 adjacent DNA bases (guanine and adenine), hence disrupting DNA replication and transcription (15). Thus, the purpose of this study was to investigate the effects of genistein and oxaliplatin (either alone or in combination) during experimentally DMBA induced oral carcinogenesis on HBP mucosa using CD44 antibody as a CSCs marker.

\section{MATERIALS AND METHODS}

The study was performed in the Faculty of Dentistry, Alexandria University after gaining the approval of the Research Ethics Committee. It comprised 100 young pathogen free Syrian hamsters, 8 weeks old. The hamsters were obtained from Theodor Bilharz Research Institute, Cairo, Egypt. They were housed in the experimental animal unit in the Medical Technology Center, Medical Research Institute, Al-Azhar University, Assiut Branch. The hamsters were kept in show polypropylene cages; they were housed as 6 per cage, in a room with controlled temperature and humidity with 12 hours light/dark cycles. They were provided with sterilized soy-free diet comprising 16\% protein, tap water ad libitum. The materials used in this study were DMBA (Sigma, USA), genistein as a chemopreventive agent (Sigma, USA) and oxaliplatin as a chemotherapeutic agent (Mylan, USA) as well as the immunohistochemical (IHC) universal kit and the primary antibody, CD44 (abcam, Cambridge, UK).

After a week of adaptation, 4 animals were sacrificed and the normal oral mucosa was histologically examined. The remaining 96 animals were randomly divided into 2 groups as following: group I (as a control group, $n=12$ ); the right buccal pouch of these animals was painted with a heavy mineral oil only. Group II $(n=84)$; the right buccal pouches of these animals were painted 3 times a week with $0.5 \%$ DMBA mixed in a heavy mineral oil. These animals were randomly divided into 4 subgroups as following: group IIA $(\mathrm{n}=12)$; which only painted with DMBA. Group IIB ( $n=24)$; genistein was orally administrated as a suspension in distilled water (20 $\mathrm{mg} / \mathrm{kg}$ body weight/day) by gavage (11). Group IIC $(n=24)$; the animals were intraperitoneally injected with oxaliplatin dissolved in a 5\% glucose solution at a concentration of $2 \mathrm{mg} / \mathrm{ml}$ (15). Depending on animal weight, it was injected as $4 \mathrm{mg} / \mathrm{kg}$ once a week. Group IID ( $n=24)$; in which both genistein was orally administrated and oxaliplatin was injected.
After 4, 8, 12, 16, 20 and 24 weeks, 2 to 4 animals from each group were sacrificed. The right buccal pouch of each animal was opened longitudinally through the skin wall directly excised and immersed in 10\% neutral-buffered formalin for 24 hours for fixation.

The tissue specimens were subsequently trimmed, embedded in paraffin, sectioned at 4 um thickness, mounted on glass slides, stained with hematoxylin and eosin and the histological sections of the lesions from all control and test groups were studied under the light microscope. Other serial sections of 3-4 um thickness were mounted on super-frost plus-coated glass slides for IHC staining with monoclonal CD44 antibody. For this, representative slides were deparaffinized, rehydrated and immunostained by the peroxidase anti-peroxidase method. High temperature antigen unmasking technique was employed in citrate buffer pH 6.0 in microwave oven, twice for 5 min each. After rinsing in phosphate buffered saline, the sections were incubated in secondary antiserum. They were then washed in PBS and incubated in avidine-biotine complex reagents and incubated in peroxidase reaction containing $0.01 \%$ H2O2 in PBS buffer (16).

The CD44 expression was scored using an image analyzer. Its staining were enumerated by using a high magnification of $x 400$ at least 10 areas of each animal were randomly chosen to determine: the CD44 area percent containing the most intense immunostained tissues and the CD44 optical density to measure the intensity of immunoreactivity.

\section{Statistical Analysis:}

The data were collected, tabulated and statistically analyzed using the SPSS system (release 11.0 software). All results were expressed as mean \pm standard deviation (SD). Oneway ANOVA was used to analyze the data between the study groups. It was also used to analyze the mean CD44 area percent and optical density of immunohistochemical results. Post Hoc Multiple comparisons of the last significant difference (LSD) 0.05. In all statistical results, a $\mathrm{P}<0.05$ was considered significant.

\section{RESULTS}

The present experimental study was performed on 100 young golden Syrian hamsters. The skin wall of the right cheek pouch mucosa of each was retracted and the medial wall was examined carefully for the presence or absence of any pathological changes. Clinical and Histological Evaluation

The cancer incidence had a range of variation between the study groups (Figure 1) as following:

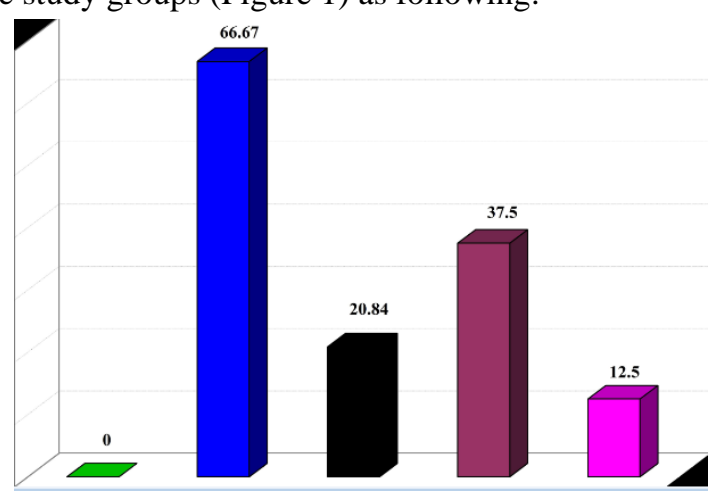

Figure 1: Relation between the study groups according to percentage of animals that developed oral cancer. 
In group I (as control group) in which, the HBP were only painted with a mineral oil, no pathological changes were developed during the study period.

In group IIA, which were treated only with DMBA, different grades of oral tumor were developed in 8 out of 12 examined animals (66.67\%). The oral lesions varied from carcinoma in situ to poorly differentiated OSCC. The difference in cancer incidence was highly statistically significant $(\mathrm{p}<0.0001)$ when comparing between group IIA and group I.

In group IIB, the hamsters were treated with DMBA concurrently with genistein $(20 \mathrm{mg} / \mathrm{kg}$ animal body weight/day) as a chemopreventive agent. All over the study, 5 out of 24 animals (20.84\%) exhibited OSCC which varied from early invasive OSCC to the moderate differentiated type. The difference in cancer incidence was highly statistically significant (p <0.0001) when comparing between group IIB and group IIA. Also, when comparing between group IIB and group I.

In group IIC, the oxaliplatin as a chemotherapeutic drug was injected $4 \mathrm{mg} / \mathrm{kg}$, once weekly after 6 weeks of painting the HBPs with DMBA. All over the study, 9 out of 24 animals (37.5\%) exhibited OSCC which varied from well to moderate differentiated type of OSCC. The difference in cancer incidence was highly statistically significant (p $<0.0001$ ) when comparing between group IIC and the previous groups; IIB, IIA and group I.

In group IID, the animals were subjected to the combined effect of the treatment with genistein and oxaliplatin during the carcinogenesis process which was induced by DMBA. All over the study, 3 out of 24 animals (12.5\%) exhibited OSCC which varied from early invasive OSCC to well differentiated type. The difference in cancer incidence was highly statistically significant $(p<0.0001)$ when comparing between group IID and the other study groups; IIC, IIB, IIA and group I.

\section{Immunohistochemical Evaluation}

Immunohistochemical evaluation was done among the study groups using CD44 monoclonal antibody as CSCs marker.

In normal HBP, CD44 immune reaction was observed, mainly in the basal and parabasal layers more than in the superficial layers of the epithelium. It showed mild immuno staining to CD44 (Figure 2). Immunohistochemical CD44 reaction was also present in some connective tissue cells (e.g. lymphocytes, fibroblasts and blood vessel walls).

The immunoreaction to CD44 in the different study groups showed variations (Figure 3-6). CD44 area percent and optical density were recorded by using an image analyzer computer system (Table 1,2).

In group I (control group), there was a positive expression of the CD44 antibody which was similar to that in the normal epithelium. The differences in the CD44 area percent and optical density were not statistically significant ( $p>0.05$ ) when compared with the normal epithelium.

In group IIA, The differences in both CD44 area percent and optical density were highly statistically significant ( $\mathrm{p}<0.0001)$ when comparing between group IIA and group I.

In group IIB, the differences in both CD44 area percent and optical density were highly statistically significant (p $<0.0001$ ) when comparing between group IIB and group IIA. The differences were not statistically significant in both the CD44 area percent and optical density when comparing between group IIB and group I.

In group IIC, the difference in CD44 area percent was highly statistically significant ( $\mathrm{p}<0.0001$ ) when comparing between group IIC and group IIA. The difference in CD44 area percent was not statistically significant when comparing between group IIC and group IIB as well as when comparing between group IIC and group I. The differences in CD44 optical density were statistically significant when comparing between group IIC and the previous groups; IIB, IIA and group I.

In group IID, the difference in CD44 area percent was highly statistically significant ( $p<0.0001$ ) when comparing between group IID and group IIA. The difference in CD44 area percent was not statistically significant when comparing between group IID and group IIC, or group IIB, or group I. The differences in CD44 optical density were statistically significant when comparing between group IID and the group IIA ( $<<0.0001)$, as well as when comparing between group IID and group IIC (p <0.0002). The differences in CD44 optical density were not statistically significant when comparing between group IID and the group IIB as well as when comparing between group IID and group I.

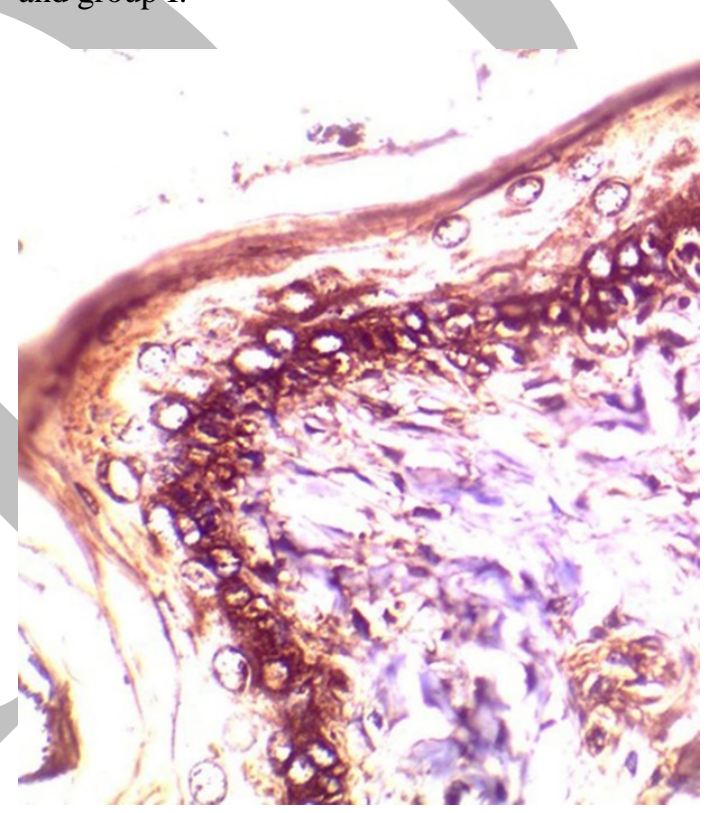

Figure 2: Normal HBP epithelial lining showing positive expression of CD44 in basal and parabasal layers. (X400).

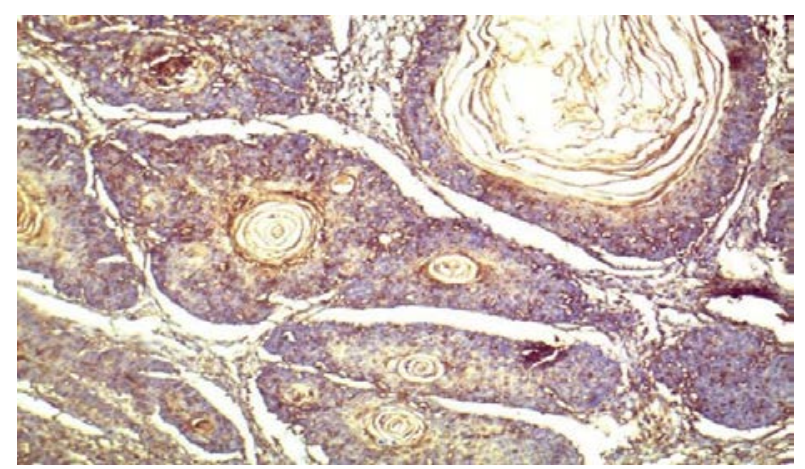

Figure 3: Well differentiated OSCC showing CD44 Expression in malignant epithelial cells (X100). 


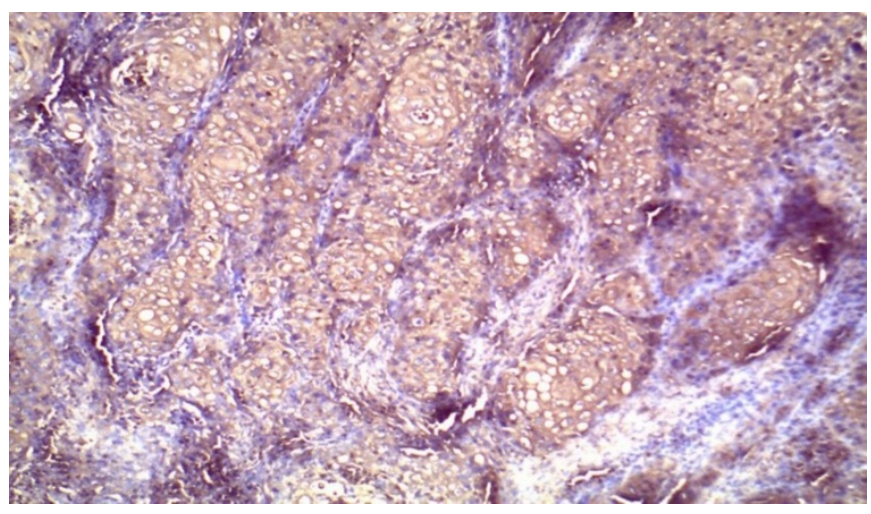

Figure 4: Moderately differentiated OSCC showing positive cytoplasmic expression of CD44 (X100).

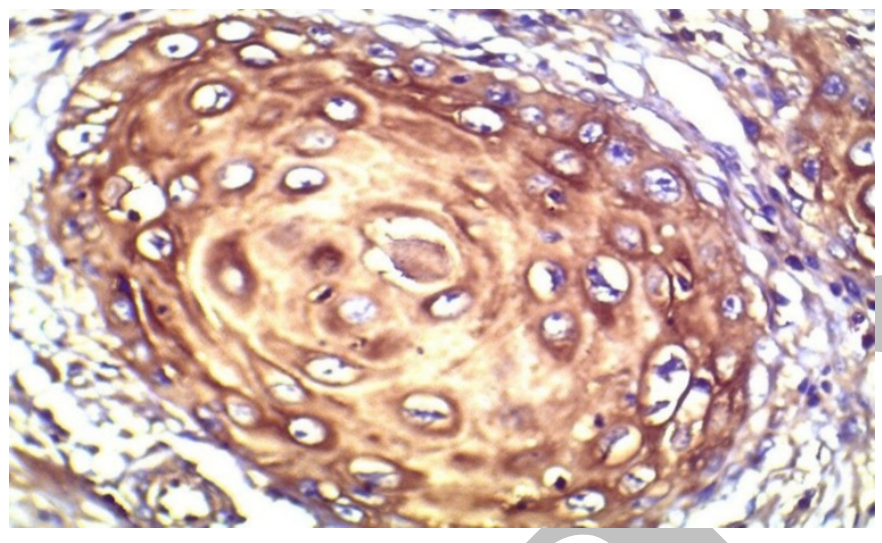

Figure 5: Moderately differentiated OSCC showing intense membranous CD44 expression in a cell nest (X400).

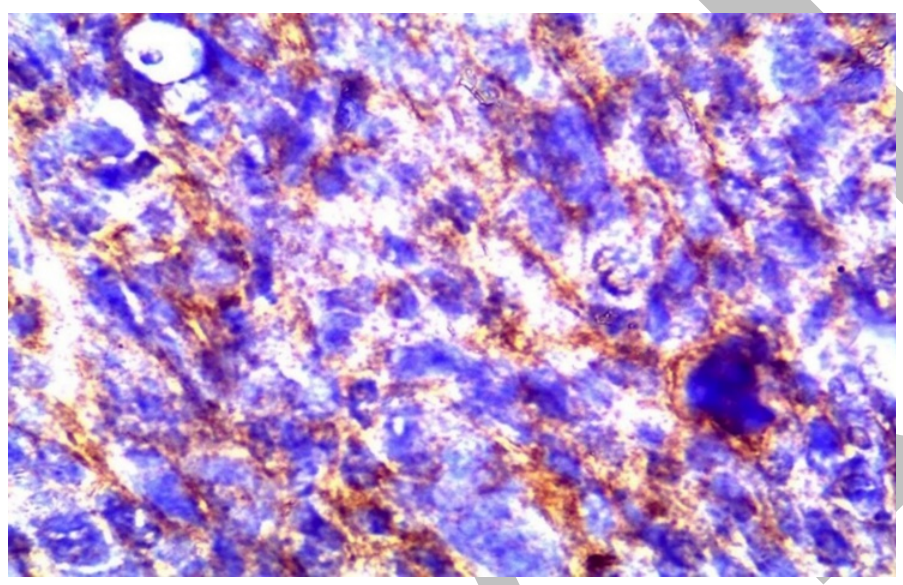

Figure 6: Poorly differentiated OSCC showing membranous CD44 expression (X1000).

Table 1: Relation between the Study Groups Concerning the CD44 Area Percent:

\begin{tabular}{|c|c|c|c|c|c|}
\hline Group & I & IIA & IIB & IIC & IID \\
\hline Min. & 19.00 & 29.10 & 21.60 & 27.90 & 26.70 \\
\hline Max. & 41.10 & 56.40 & 53.50 & 44.00 & 43.60 \\
\hline Mean & 32.10 & 43.08 & 34.80 & 35.45 & 33.80 \\
\hline SD & 6.11 & 8.74 & 6.48 & 4.54 & 3.87 \\
\hline
\end{tabular}

Table 2: CD44 Optical Density in the different study groups.

\begin{tabular}{|c|c|c|c|c|c|}
\hline Group & I & IIA & IIB & IIC & IID \\
\hline Min. & 37.70 & 42.40 & 35.50 & 38.70 & 33.60 \\
\hline Max. & 59.90 & 92.70 & 87.60 & 86.60 & 66.90 \\
\hline Mean & 48.61 & 66.27 & 50.25 & 57.14 & 47.49 \\
\hline SD. & 6.79 & 13.85 & 10.21 & 12.42 & 8.10 \\
\hline
\end{tabular}

\section{DISCUSSION}

In the current study, the reduction in the carcinogenesis process was highly statistically significant in animals that received genistein orally, with a daily dose of $20 \mathrm{mg} / \mathrm{kg}$, (group IIB) compared to the animals that were only painted by DMBA (group IIA). In agreement with the results of the present study, Ghaemi et al. (17) suggested that orally administrated genistein, $20 \mathrm{mg} / \mathrm{kg}$ by gavage, exerted its effects in mouse model of cervical cancer thereby inducing protective antitumor immunity. In addition, Pugalendhi et al. (18) indicated that the oral pretreatment of genistein (20 $\mathrm{mg} / \mathrm{kg}$ body weight/day), reversed the frequency of the carcinogenesis in DMBA treated rats. Consistent with the previous findings Johnson et al. (19) reported that the treatment with the isoflavones, genistein could be useful as chemopreventive agent and may be employed as chemotherapy for oral carcinomas. The results of this study as well as those discussed above strongly supported that genistein as a chemopreventive agent exerts an antiproliferative effect on a variety of cancer cell types.

On the other hand, Myoung et al. (20) did not find any inhibitory effect for genistein on the transplanted human OSCC. In their study, the rapidly growing transplantable mouse tumor model was employed as a solid localized tumor in the subcutaneous space. This kind of tumor models usually contain high proportions of newly formed immature blood vessels that appear to weaken the effects of most antiangiogenic drugs. In addition, the administration of genistein after the initiation stage of the carcinogenesis process and at a dosage relevant to the real-life consumption pattern (0.3 to $1 \mathrm{mg} / \mathrm{kg})$ in a study done by Yong et al. (21) did not find any preventive effect of genistein on DMBA induced oral HBP carcinogenesis. The variation in the dose concentrations of genistein may exert different effects on the oral carcinogenesis process. The duration of genistein administration may affect the metabolism, bioavailability and its biological action.

In the present research, oxaliplatin demonstrated effectiveness and tolerance in reducing the DMBA carcinogenesis process in a dose of $4 \mathrm{mg} / \mathrm{kg}$ once weekly. To the best of our knowledge, this was the first time that oxaliplatin was used as a line of treatment during oral HBP carcinogenesis. In this study, the difference in cancer incidence was highly statistically significant when comparing between group IIC and group IIA.

It was proved that oxaliplatin is one of the most active drugs for the treatment of colorectal cancer especially its metastatic type (22). Moreover, adenoid cystic carcinoma of the salivary glands demonstrated an objective response with oxaliplatin based chemotherapy (23). Hung et al. (24) studied the differential resistance of platinum based drugs to OSCC, they implicated that oxaliplatin may be a treatment option especially for OSCC. Furthermore, Shen et 
al. (25) reported that oxaliplatin was a promising agent for chemotherapy in treating esophageal SCC.

In contrast, Lim et al. (26) suggested that the oxaliplatin did not lead to better efficacy in node-positive esophageal SCC patients. A possible explanation for this negative result could be that over $50 \%$ of the enrolled patients in the mentioned study had advanced nodal disease. So that, the treatment with chemotherapy alone was not probably sufficient to control the recurrence. Moreover, Yang et al. (26) reported that the time factor should be considered when treating the OSCC patients with oxaliplatin in order to achieve a better efficacy, reduce the adverse reactions and improve the survival time.

The purpose of combining both genistein and oxaliplatin in group IID was to reduce the carcinogenic effect induced by DMBA, which was highly statistically significant when compared to the group treated only with DMBA (group IIA). The present results indicate that the combined use of a natural chemopreventive agent and a chemotherapeutic drug is more effective in reducing the carcinogenesis process than using each one alone.

In agreement with the present research results, genistein may be used for achieving a better treatment outcome in patients diagnosed with SCC with platinum resistant (28). In addition, Banerjee et al. (29) provided preclinical evidence that genistein appeared to be a novel approach for the treatment of pancreatic cancer with oxaliplatin. Furthermore, a study done by Pintova et al. (30) suggested that the effect of the soy isoflavones genistein and oxaliplatin inhibited the carcinogenesis pathway in colorectal cancer cells.

The current study results demonstrated that CD44 expression had a key role in tumor aggressiveness. A marked increase in the CD44 area percent and optical density in the examined tissues of hamsters which were only treated with DMBA (group IIA) were reported. They demonstrated highly statistically significant when compared with the examined tissues of the control animals group (group I).

In agreement with the present study results, Judd et al. (31) reported that the decrease in CD44 expression led to delay in the tumor growth. In addition, Paulis et al. (32) concluded that CD44 expression increased the aggressiveness of tumor cells behavior. Furthermore, Rajarajan et al. (33) demonstrated an increase in CD44 expression in head and neck cancer relative to its expression in other cancer types. This goes in line with the results of the present research.

On the other hand, Margaritescu et al. (34) showed a limited usefulness of CD44 expression in identifying the CSCs in OSCCs. Moreover, Krump et al. (35) demonstrated that there are no significant differences in CD44 expression between the different tumor grades in the oral cavity. This shortening in the role of the CD44 may be due to improper selection of the examined tissues which may have massive areas of inflammation leading to a false results in expression of the CD44. Moreover, the difference in the sorting techniques, than the IHC, for identification the CD44 expression in tissue as Western blotting and flow cytometric assessments may demonstrate different sorting results.

The present study reported that the differences in both the CD44 area percent and optical density were highly statistically significant when comparing between group IIA and group IIB as well as group IIC. Moreover, the differences in both the CD44 expression parameters were highly statistically significant when comparing between group IIA and the group which was given both genistein and oxaliplatin (group IID). This supports the antitumor chemopreventive role of genistein and the putative chemotherapeutic activity of the oxaliplatin during DMBA induced carcinogenesis process in the HBPs performed in the present research.

Comparable results obtained by Dandawate et al. (36) demonstrated that the natural phytochemicals as genistein may serve as a novel therapeutic agent for breast cancer as it has a role in targeting the CSCs. In addition, Sak et al. (37) reported that the flavonoids agents as genistein served as attractive anticancer agents by eliminating the roots of cancers. This goes in line with the results of the present work. However, Cao et al. (38) reported that the genistein provided a proper chemopreventive action in gastric CSCs when given in combination with other drugs than applied alone.

This was in accordance with the results of the present research, which confirmed the proper chemotherapeutic effect of oxaliplatin in the CSCs. Dallas et al. (39) identified that the colorectal CSCs showed a decrease in cellular proliferation when treated with oxaliplatin. On the other hand, Huang et al. (40) found that the oxaliplatin needs modulation in its action pathway to overcome the chemoresistance of CSCs cells in a clinical setting of colorectal cancer. This shortening may be due to the great variation in the tumor grades and activities during the previous clinical study than in the presented experimental one.

\section{CONCLUSION}

Based on results of the present study, genistein provides chemoprevention role and oxaliplatin produce chemotherapeutic activity in developed oral cancer. The combined action of both agents was better than each one alone. The CD44 expression gives an idea about the aggressiveness of tumor and the effects of the anticancer drug on CSCs activity and provided new strategy for evaluating of the chemotherapeutic action of anticancer drugs.

\section{CONFLICT OF INTEREST}

The authors declare that they have no conflicts of interest.

\section{REFERENCES}

1. Mignogna MD, Fedele S, Lo Russo L. The world cancer report and the burden of oral cancer. Eur J Cancer Prev. 2004;13:139-42.

2. Simard EP, Torre LA, Jemal A. International trends in head and neck cancer incidence rates: differences by country, sex and anatomic site. Oral Oncol. 2014;50:387-403.

3. Nagini S, Kowshik J. The Hamster Buccal Pouch Model of Oral Carcinogenesis. Methods Mol Biol. 2016;1422:341-50.

4. Suresh K, Manoharan S, Vijayaanand MA, Sugunadevi G. Chemopreventive and antioxidant efficacy of (6)paradol in 7,12-dimethylbenz(a)anthracene induced hamster buccal pouch carcinogenesis. Pharmacol Rep. 2010;62:1178-85. 
5. Al-Hajj M, Clarke MF. Self-renewal and solid tumor stem cells. Oncogene. 2004;23:7274-82.

6. Tai MH, Chang CC, Kiupel M, Webster JD, Olson LK, Trosko JE. Oct4 expression in adult human stem cells: evidence in support of the stem cell theory of carcinogenesis. Carcinogenesis. 2005;26:495-502.

7. Klonisch T, Wiechec E, Hombach-Klonisch S, Ande SR, Wesselborg S, Schulze-Osthoff K, et al. Cancer stem cell markers in common cancers-therapeutic implications. Trends Mol Med. 2008;14:450-60.

8. Misra S, Hascall VC, Berger FG, Markwald RR, Ghatak S. Hyaluronan, CD44, and cyclooxygenase-2 in colon cancer. Connect Tissue Res. 2008;49:219-24.

9. Karikas GA. Anticancer and chemopreventing natural products: some biochemical and therapeutic aspects. J BUON. 2010;15:627-38.

10. Rajkamal G, Suresh K, Sugunadevi G, Vijayaanand MA, Rajalingam K. Evaluation of chemopreventive effects of Thymoquinone on cell surface glycoconjugates and cytokeratin expression during DMBA induced hamster buccal pouch carcinogenesis. BMB Rep. 2010;43:664-9.

11. Pugalendhi P, Manoharan S, Suresh K, Baskaran N. Genistein and daidzein, in combination, protect cellular integrity during 7,12-dimethylbenz[a]anthracene (DMBA) induced mammary carcinogenesis in SpragueDawley rats. N Afr J Tradit Complement Altern Med. 2011;8:91-7.

12. Huang W, Wan C, Luo Q, Huang Z, Luo Q. Genisteininhibited cancer stem cell-like properties and reduced chemoresistance of gastric cancer. Int $\mathrm{J}$ Mol Sci. 2014;15:3432-43.

13. Karikas GA. Chemoprevention molecular and biochemical mechanisms involved in cancer control and management. Heal Scien J. 2011;5:149-56.

14. American Cancer Society. Chemotherapy drugs: how they work. Last Medical Review. 2015;1:1-17.

15. Baker DE. Oxaliplatin: a new drug for the treatment of metastatic carcinoma of the colon or rectum. Rev Gastroenterol Disord. 2003;3:31-8.

16. Hema K, Rao K, Devi HU, Priya N, Smitha T, Sheethal $\mathrm{H}$. Immunohistochemical study of CD44s expression in oral squamous cell carcinoma-its correlation with prognostic parameters. J Oral Maxillofac Pathol. 2014;18:162-8.

17. Ghaemi A, Soleimanjahi H, Razeghi S, Gorji A, Tabaraei A, Moradi A. Genistein induces a protective immunomodulatory effect in a mouse model of cervical cancer. Iran J Immunol. 2012;9:119-27.

18. Pugalendhi P, Manoharan S, Panjamurthy K, Balakrishnan S, Nirmal MR. Antigenotoxic effect of genistein against 7,12-dimethylbenz[a]anthracene induced genotoxicity in bone marrow cells of female Wistar rats. Pharmacol Rep. 2009;61:296-303.

19. Johnson TL, Lai MB, Lai JC, Bhushan A. Inhibition of cell proliferation and MAP kinase and Akt pathways in oral squamous cell carcinoma by genistein and biochanin A. Evid Based Complement Alternat Med. 2010;7:351-8.

20. Myoung $\mathrm{H}$, Hong SP, Yun PY, Lee JH, Kim MJ. Anticancer effect of genistein in oral squamous cell carcinoma with respect to angiogenesis and in vitro invasion. Cancer Sci. 2003;94:215-20.
21. Yang Y, Zhou ZT, Ge JP. Effect of genistein on DMBA-induced oral carcinogenesis in hamster. Carcinogenesis. 2006;27:578-83.

22. Burz C, Berindan-Neagoe IB, Balacescu O, Tanaselia C, Ursu M, Gog A, et al. Clinical and pharmackinetics study of oxaliplatin in colon cancer patients. J gastrointestin liver Dis. 2009;18:39-43.

23. De Dosso S, Mazzucchelli L, Ghielmini M, Saletti P. Response to oxaliplatin with cetuximab in minor salivary gland adenoid cystic carcinoma. Tumori. 2009;95:378-81.

24. Inadomi K, Kusaba $H$, Matsushita $Y$, Tanaka R, Mitsugi K, Arimizu K, et al. Efficacy and safety analysis of oxaliplatin-based chemotherapy for advanced gastric cancer. Anticancer Res. 2017;37:2663-71.

25. Shen Z, Xu L, Li J, Zhang N. Capilliposide C sensitizes esophageal squamous carcinoma cells to oxaliplatin by inducing apoptosis through the PI3K/Akt/mTOR pathway. Med Sci Monit. 2017;23:2096-103.

26. Fakhrian K, Ordu AD, Haller B, Theisen J, Lordick F, Bisof V, et al. Cisplatin- vs. oxaliplatin-based radiosensitizing chemotherapy for squamous cell carcinoma of the esophagus: a comparison of two preoperative radiochemotherapy regimens. Strahlenther Onkol. 2014;190:987-92.

27. Yang K, Zhao N, Zhao D, Chen D, Li Y. The drug efficacy and adverse reactions in a mouse model of oral squamous cell carcinoma treated with oxaliplatin at different time points during a day. Drug Des Devel Ther. 2013;7:511-7.

28. Ali S, Varghese L, Pereira L, Tulunay-Ugur OE, Kucuk $\mathrm{O}$, Carey TE, et al. Sensitization of squamous cell carcinoma to cisplatin induced killing by natural agents. Cancer Lett. 2009;278:201-9.

29. Banerjee S, Kong D, Azmi AS, Wang Z, Ahmad A, Sethi $S$, et al. Restoring sensitivity to oxaliplatin by a novel approach in gemcitabine-resistant pancreatic cancer cells in vitro and in vivo. Int $\mathrm{J}$ Cancer. 2011;128:1240-50.

30. Pintova S, Planutis K, Planutiene M, Holcombe RF. ME-143 is superior to genistein in suppression of WNT signaling in colon cancer cells. Anticancer Res. 2017;37:1647-53.

31. Judd NP, Winkler AE, Murillo-Sauca O, Brotman JJ, Law JH, Lewis JS Jr, et al. ERK1/2 regulation of CD44 modulates oral cancer aggressiveness. Cancer Res. 2012;72:365-74.

32. Paulis YW, Huijbers EJ, van der Schaft DW, Soetekouw PM, Pauwels P, Tjan-Heijnen VC, et al. CD44 enhances tumor aggressiveness by promoting tumor cell plasticity. Oncotarget. 2015;6:19634-46.

33. Rajarajan A, Stokes A, Bloor BK, Ceder R, Desai H, Grafström RC, et al. CD44 expression in oropharyngeal carcinoma tissues and cell lines. PLoS One. 2012;7:e28776.

34. Margaritescu C, Pirici D, Simionescu C, Stepan A. The utility of CD44, CD117 and CD133 in identification of cancer stem cells (CSC) in oral squamous cell carcinomas (OSCC). Rom J Morphol Embryol. 2011;52:985-93.

35. Krump M, Ehrmann J. Differences in CD44s expression in HNSCC tumors of different areas within 
the oral cavity. Biomed Pap Med Fac Univ Palacky Olomouc Czech Repub. 2013;157:280-3.

36. Dandawate PR, Subramaniam D, Jensen RA, Anant S. Targeting cancer stem cells and signaling pathways by phytochemicals: Novel approach for breast cancer therapy. Semin Cancer Biol. 2016;40-41:192-208.

37. Sak K, Everaus H. Role of flavonoids in future anticancer therapy by eliminating the cancer stem cells. Curr Stem Cell Res Ther. 2015;10:271-82.

38. Cao X, Ren K, Song Z, Li D, Quan M, Zheng Y, et al. 7-Difluoromethoxyl-5,4'-di-n-octyl genistein inhibits the stem-like characteristics of gastric cancer stem-like cells and reverses the phenotype of epithelialmesenchymal transition in gastric cancer cells. Oncol Rep. 2016;36:1157-65.

39. Dallas NA, Xia L, Fan F, Gray MJ, Gaur P, van Buren $\mathrm{G}$ 2nd, et al. Chemoresistant colorectal cancer cells, the cancer stem cell phenotype, and increased sensitivity to insulin-like growth factor-I receptor inhibition. Cancer Res. 2009;69:1951-7.

40. Huang R, Wang G, Song Y, Tang Q, You Q, Liu Z, et al. Colorectal cancer stem cell and chemoresistant colorectal cancer cell phenotypes and increased sensitivity to Notch pathway inhibitor. Mol Med Rep. 2015;12:2417-24. 